\title{
Meta-Analysis of Homogeneous Subgroups Reveals Association between PDE4D Gene Variants and Ischemic Stroke
}

\author{
Dankyu Yoon $^{\mathrm{a}}$ Sue K. Park ${ }^{\mathrm{c}-\mathrm{e}}$ Daehee Kang ${ }^{\mathrm{c}-\mathrm{e}}$ Taesung Park $^{\mathrm{b}}$ Ji Wan Park \\ ${ }^{a}$ Interdisciplinary Program in Bioinformatics, and ${ }^{b}$ Department of Statistics, College of Natural Science, \\ Seoul National University, ' Department of Preventive Medicine, Seoul National University College of \\ Medicine, ${ }^{\mathrm{d} C a n c e r}$ Research Institute, Seoul National University, and ${ }^{\mathrm{e}}$ Department of Biomedical Science, \\ Seoul National University Graduate School, Seoul, and f Department of Medical Genetics, Hallym University College \\ of Medicine, Chuncheon, Republic of Korea
}

\section{Key Words}

Phosphodiesterase 4D $\cdot$ Ischemic stroke $\cdot$ Meta-analysis • Single nucleotide polymorphism $\cdot$ Genetic association study

\begin{abstract}
Background: An Icelandic study showed a significant positive association between phosphodiesterase 4D (PDE4D) gene variants and stroke. However, subsequent studies reported conflicting results, possibly due to small sample sizes and the heterogeneity of the studies. Method: We performed a meta-analysis on 6 SNPs of the PDE4D gene to investigate the association between this gene and ischemic stroke by integrating the results of previous studies, comprising 11,834 cases and 15,233 controls. A pooled genotypic odds ratio (OR) for each SNP was determined under 3 genetic models (i.e. dominant, recessive, and codominant) using both fixed-and random-effects models with consideration for heterogeneity and publication bias across studies. Results: Among the SNPs included in this study, SNP56 (rs702553) showed the most significant association with ischemic stroke in a meta-analysis comprised of 7 homogenous studies. The overall OR of the TT genotype compared to the
\end{abstract}

AA genotype was 1.29 (95\% Cl 1.03-1.61; $p=0.022)$. For SNP83 (rs966221), a protective effect of the ancestral allele T was observed only in Asian populations (ORTT $0.79,95 \% \mathrm{Cl}$ $0.69-0.90 ; p=0.0005$ ). This meta-analysis revealed a significant association of PDE4D gene variants with the risk of ischemic stroke, and further investigations are warranted to evaluate possible ethnic-specific effects.

Copyright $\odot 2011$ S. Karger AG, Basel

\section{Introduction}

According to the World Health Organization (WHO), stroke is a leading cause of death and disability and was responsible for 5.7 million deaths worldwide in 2005 [1]. Ischemic stroke, the most common subtype of stroke, accounts for $85 \%$ of all strokes [2]. Previous genetic epidemiological studies provided substantial evidence that susceptibility to ischemic stroke is determined by a complex interplay of genetic and environmental factors. Conventional risk factors include nonmodifiable factors (i.e. age, gender, and ethnicity), modifiable environmental factors (i.e. cigarette smoking and a high-calorie diet), and disease-related phenotypes (i.e. homocysteine con-

\section{KARGER}

Fax +41613061234 E-Mail karger@karger.ch www.karger.com
(C) 2011 S. Karger AG, Basel

0251-5350/11/0364-0213\$38.00/0

Accessible online at:

www.karger.com/ned
Dr. Ji Wan Park

Department of Medical Genetic

Hallym University College of Medicine, 39 Hallymdaehak-gil

Chuncheon, Gangwon-do 200-702 (Republic of Korea)

Tel. +82 33248 2691, E-Mail jwpark@ hallym.ac.kr 
centration, hypertension, diabetes, etc.) [3]. Genetic predisposition may differ depending on the age, gender, ethnicity, and stroke subtype (i.e. small-vessel stroke, largevessel stroke, and cardio-embolic stroke) of the study population [4-6]. There are no published populationbased data on heritability for ischemic stroke. Alternatively, heritability estimates for such intermediate phenotypes of stroke as cerebral white matter lesions and intima-media thickness were in the ranges of 55-70 and $30-60 \%$, respectively $[7,8]$.

Phosphodiesterase 4D (PDE4D) (MIM 600129) is a very large gene spanning $>1.5 \mathrm{Mb}$ on chromosomal region $5 \mathrm{q} 12$ with 22 exons, 8 splice variants, and several hundred SNPs $[9,10]$. Gretarsdottier et al. [10] of the Iceland DeCODE study reported evidence for the linkage of a susceptibility gene for ischemic stroke to the $5 \mathrm{q} 13$ chromosomal region in 2002 and subsequently found a significant association between the PDE $4 D$ gene and ischemic stroke in 2003. Thus far, more than 20 studies have attempted to replicate the findings of the Icelandic study, but some of these studies have reported conflicting results [11-13]. Even 3 publications that conducted analyses identical to that of the initial study using the same markers in the same stroke subtype did not replicate the original findings of Gretarsdottir et al. [2, 4, 10-12]. There could be a variety of explanations for these discrepancies, including insufficient statistical power due to a small sample size, nonstandardized adjustments for modifiable and nonmodifiable risk factors for stroke, and different analytical methods, ethnic populations, diagnostic criteria, and stroke subtypes applied [14]. Some of these problems can be resolved through meta-analysis, an efficient method to increase statistical power by pooling samples obtained from independent studies [15].

However, meta-analysis on the association between the PDE $4 D$ gene and stroke has proven difficult to perform because of the lack of accumulated information on each SNP, different SNPs selected as target markers across studies, and the heterogeneity of the phenotypes examined among studies [2]. To date, 5 meta-analyses have been reported with respect to the relationship between the PDE4D gene and stroke. Staton et al. [13] (2006) included 9 case-control studies composed of 3,808 patients with stroke and 4,377 controls without consideration of subtype. Three SNPs, i.e. $41(\mathrm{p}=0.003), 83(\mathrm{p}=0.003)$, and 87 (pooled $p=0.002$ ), showed a significant association with overall stroke even after adjustment for multiple comparisons, although heterogeneity was detected using the Q test ( $\mathrm{p}<0.1)$. Lövkvist et al. [16] (2008) analyzed only SNP45 in their meta-analyses of the random- effects model which was comprised of 13 studies $(6,221$ Caucasian cases and 6,750 controls) and showed no significant overall effect of SNP45 on ischemic stroke. The heterogeneity test suggests that the odds ratios (ORs) of the 13 studies are more heterogeneous than expected by random variation alone $(\mathrm{p}=0.042)$. Bevan et al. [17] (2008) performed a series of meta-analyses of 16 studies only under the fixed-effects model. The associations of 6 individual SNPs (i.e. SNP 26, 45, 56, 83, 87, and 89) were tested for both carriers (i.e. under the dominant model) and homozygotes (i.e. under the recessive model). None of the meta-analyses for ischemic stroke cases could replicate the statistical significance of an association reported in previous studies. Recently, 2 ethnic specific metaanalyses were published. Domingues-Monatanari et al. [18] (2010) reported that none of the SNPs in the PDE4D gene was associated with ischemic stroke in the Iberian population. However, Xu et al. [19] (2010) found an association between SNP83 and ischemic cerebral infarction in Asian populations.

Nonetheless, the PDE4D gene encoding an enzyme that selectively degrades cAMP appears to be a potential candidate gene for stroke through its enzyme activity in regulating the response of human tissues to injury or infections $[20,21]$. The significant heterogeneity of the studies being combined might be responsible for the failure of these meta-analyses to replicate previous findings. Therefore, we performed a meta-analysis on 6 SNPs (i.e. SNPs $26,45,56,83,87$, and 89) with consideration of heterogeneity and bias being introduced in the studies to evaluate the effect of the PDE $4 D$ gene variants in the occurrence of ischemic stroke.

\section{Materials and Methods}

\section{Systematic Review of Studies}

Using a systematic search of the MEDLINE electronic database (PubMed, http://www.ncbi.nlm.nih.gov/sites/entrez/) and SCOPUS (http://www.scopus.com/scopus/home.url) from January 2003 to August 2010, we identified all studies on the association of PDE4D gene variants with ischemic stroke. The terms used for the search were 'ischemic (ischaemic) stroke genotype cohort', 'phosphodiesterase 4D ischemic (ischaemic) stroke genotype', 'cAMP-specific (PDE4D) ischemic (ischaemic) stroke genotype', and 'ischemic (ischaemic) stroke PDE4D'. Studies were included in our meta-analysis if the following criteria were fulfilled: firstly, an association between ischemic stroke and SNPs near or in the PDE4D gene was examined; secondly, the study was a prospective cohort, nested case-control, or case-control study, and thirdly, studies that provided genotype or allele frequencies on common SNPs among independent studies were included. In case of lack of genotype frequency information from papers, we contacted 
Table 1. $P D E 4 D$ gene variants evaluated for an association with ischemic stroke in previous studies

\begin{tabular}{|c|c|c|c|}
\hline Study & Ethnicity & $\begin{array}{l}\text { Number of } \\
\text { cases/controls }\end{array}$ & $\mathrm{SNP}^{\mathrm{a}}$ \\
\hline Gretarsdottir et al. [10] & Icelandic & $864 / 908$ & SNPs $26,45,56,83,87,89$ \\
\hline Bevan et al. [11] & English & $737 / 933$ & SNPs $26,45,87$ \\
\hline Lohmussaar et al. [12] & German & $639 / 736$ & SNPs $26,45,87$ \\
\hline Meschia et al. [39] & European-American, others & $377 / 263$ & SNPs $45,56,83$ \\
\hline Nakayama et al. [28] & Japanese & $208 / 270$ & SNP83 \\
\hline Nilsson-Ardnor et al. [43] & Swedish & $275 / 550$ & SNP45 \\
\hline Saleheen et al. [30] & Pakistani & $200 / 250$ & SNPs 83,87 \\
\hline van Rijn et al. [42] & Dutch & $91 / 200$ & SNPs 45,83 \\
\hline Woo et al. [40] & European-American, African-American & $357 / 303$ & SNPs $56,83,87,89$ \\
\hline Brophy et al. [37] & European-American & $248 / 560$ & SNPs $26,45,56$ \\
\hline Kuhlenbaumer et al. [41] & German & $1,181 / 1,569$ & SNPs $45,56,83,87,89$ \\
\hline Song et al. [36] & European-American, African-American & $224 / 211$ & SNPs $45,83,89$ \\
\hline Staton et al. [13] & Australian & $151 / 164$ & SNPs $45,56,83,87,89$ \\
\hline Zee et al. [38] & European-American & $259 / 259$ & SNPs $26,45,56$ \\
\hline Kostulas et al. [44] & Swedish & $524 / 751$ & SNP45 \\
\hline Fidani et al. [45] & Greek & $97 / 102$ & SNP45 \\
\hline Lin et al. [31] & Taiwanese & $190 / 211$ & SNPs $56,83,87$ \\
\hline Banerjee et al. [32] & Indian & $176 / 212$ & SNP83 \\
\hline Lövkvist et al. [16] & Swedish & $932 / 396$ & SNPs 45,87 \\
\hline Xue et al. [34] & Chinese & $639 / 887$ & SNPs 83,87 \\
\hline Matsushita et al. [29] ${ }^{\mathrm{b}}$ & Japanese & $2,890 / 4,412$ & SNPs $56,83,87,89$ \\
\hline Sun et al. [35] & Chinese & $649 / 761$ & SNPs $56,83,87$ \\
\hline Munshi et al. [33] & Indian & $250 / 250$ & SNP83 \\
\hline
\end{tabular}

a SNPs included in each study among the 6 SNPs that were analyzed in the Icelandic study by Gretarsdottir et al. [10].

${ }^{\mathrm{b}}$ Included 2 case-control studies and 1 cohort study.

each author to obtain the genotype data. Studies were excluded if they were cross-sectional studies or clinical trials, and studies were limited to human studies published in the English language. A total of 23 studies were included in the subsequent meta-analysis from a total of 168 independent references identified through the systematic searches (fig. 1).

\section{Statistical Analysis}

Data were analyzed using the meta-analysis package, 'meta', implemented in the statistical software environment R. (http:// www.r-project.org/). Meta-analyses were carried out for the 6 genetic markers, i.e. SNP26 (rs40512), SNP45 (rs12188950), SNP56 (rs702553), SNP83 (rs966221), SNP87 (rs2910829), and SNP89 (rs1396476), for which data were available in at least 2 different studies. Our analyses were based on the genotypic effect under 3 genetic models (i.e. dominant, recessive, and codominant). We calculated pooled ORs with 95\% confidence intervals (CI) for each of the 6 SNPs using both fixed-and random-effects models. We applied the Mantel-Haenszel method to estimate the fixedeffects summary ORs and the DerSimonian and Laird method for the summary ORs under the random-effects model [22, 23].

The degree of heterogeneity among the study results was assessed using the $\mathrm{I}^{2}$ statistic, and the significance of heterogeneity for each meta-analysis was evaluated using the $\chi^{2}$ test at $\mathrm{p}<0.01$ [23-25]. In the presence of significant heterogeneity, we per-

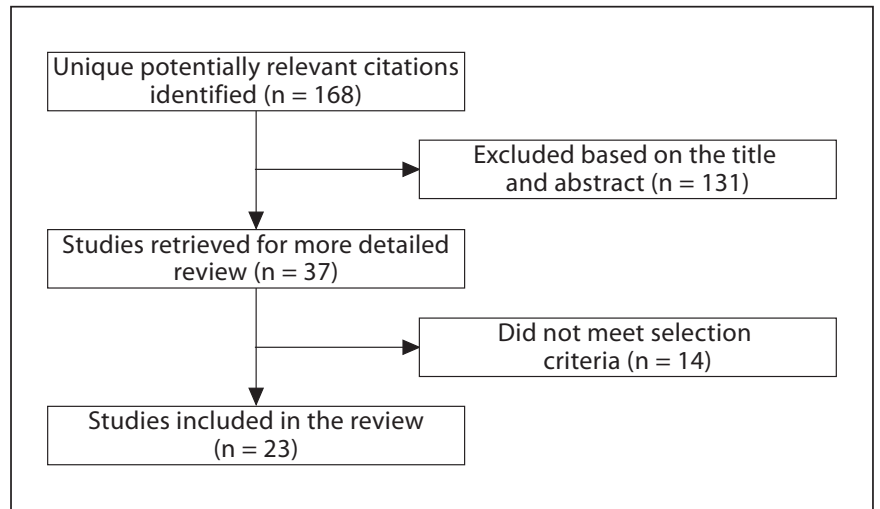

Fig. 1. Schematic flow chart depicting the selection of studies for meta-analysis.

formed the sensitivity analysis using sequential algorithms to explore the source of heterogeneity and to achieve homogeneity by removing one heterogeneous study at a time [26]. Since amongstudy variability may influence the conclusions of a meta-analysis (for instance, the effects in opposite directions across studies due 
Table 2. Characteristics of 23 studies reporting an association between $P D E 4 D$ gene variants and ischemic stroke

\begin{tabular}{|c|c|c|c|c|c|}
\hline \multirow[t]{2}{*}{ Study } & \multirow[t]{2}{*}{ Study design } & \multicolumn{3}{|l|}{ Cases/controls } & \multirow[t]{2}{*}{ Adjustment variables } \\
\hline & & selection & mean age, years & female, $\%$ & \\
\hline Gretarsdottir et al. [10] & Cohort & Hospital/population & NA & NA & NA \\
\hline Bevan et al. [11] & Case-control & Hospital/population & $65 \pm 12.5 / 65 \pm 8.9$ & $41.0 / 43.0$ & Age, sex, smoking, DM, HT, HCL \\
\hline Lohmussaar et al. [12] & Case-control & Hospital/population & $65 \pm 18.2 / 62 \pm 11.7$ & $37.7 / 39.3$ & NA \\
\hline Meschia et al. [39] & Case-control, cohort & $\begin{array}{l}\text { Hospital/spouses, } \\
\text { friends }\end{array}$ & $64.8 \pm 15.0 / 60 \pm 14.7$ & $46.4 / 62.0$ & $\begin{array}{l}\text { Age, sex, race, BMI, family history, } \\
\text { smoking, etc. }\end{array}$ \\
\hline Nakayama et al. [28] & Case-control & Hospital/hospital & $66 \pm 12.4 / 66.1 \pm 5.8$ & $39.4 / 47.8$ & NA \\
\hline Nilsson-Ardnor et al. [43] & Nested case-control & Population/population & $55 \pm 8.3$ & 42.9 & Matching for sex, age, cohort, etc. \\
\hline Saleheen et al. [30] & Case-control & Hospital/population & $62.4 \pm 12.4 / 54.1 \pm 8.9$ & $70.5 / 74.0$ & Age, sex, DM, HT \\
\hline van Rijn et al. [42] & Case-control & Population/population & $64.1 \pm 12.2 / 56.8 \pm 11.6$ & $40.7 / 60.0$ & Age, sex \\
\hline Woo et al. [40] & Case-control & Hospital/population & $69 / 68$ & $56.3 / 55.8$ & $\begin{array}{l}\text { BMI, HT, DM, smoking, family } \\
\text { history, etc. }\end{array}$ \\
\hline Brophy et al. [37] & Nested case-control & Hospital/hospital & $73.9 \pm 5.9 / 70.3 \pm 4.5$ & Female only & Age, weight, smoking, DM \\
\hline Kuhlenbaumer et al. [41] & Cohort & Hospital/hospital & $66.9 \pm 14.6 / 55.9 \pm 13.7$ & $46.4 / 51.2$ & Age, sex, DM, HT, HCL \\
\hline Song et al. [36] & Case-control & Hospital/population & $41.7 / 39.6$ & Female only & Age \\
\hline Staton et al. [13] & Case-control & Hospital/population & $67.3 \pm 11.7 / 66.1 \pm 11.8$ & $33.8 / 37.2$ & NA \\
\hline Zee et al. [38] & Nested case-control & Prospective cohort & $62.1 \pm 0.5 / 61.7 \pm 0.5$ & Male only & $\begin{array}{l}\text { Matching for age, smoking, length of } \\
\text { follow-up }\end{array}$ \\
\hline Kostulas et al. [44] & Case-control & Hospital/population & $69.7 \pm 11.4 / 46.8 \pm 14.9$ & $43.9 / 41.0$ & NA \\
\hline Fidani et al. [45] & Cohort & Hospital/population & $75.8 \pm 6.9 / 72.7 \pm 6.1$ & $48.5 / 49.0$ & NA \\
\hline Lin et al. [31] & Case-control & Hospital/population & NA & NA & Smoking, HT, DM \\
\hline Banerjee et al. [32] & Case-control & Hospital/population & $58.6 \pm 14.2 / 57.4 \pm 8.8$ & $35.8 / 32.5$ & Age, sex, smoking, HT, DM \\
\hline Lövkvist et al. [16] & & Prospective cohort & $73.6 \pm 11.5 / 73.6 \pm 11.9$ & $44 / 43.2$ & $\begin{array}{l}\text { Matching for age, sex, HT, DM, HD, } \\
\text { smoking }\end{array}$ \\
\hline Xue et al. [34] & Case-control & Hospital/population & $60.8 \pm 9.2 / 60.7 \pm 8.2$ & $37.4 / 42.4$ & $\begin{array}{l}\text { Age, sex, BMI, SBP, DBP, glucose, } \\
\text { HDL, etc. }\end{array}$ \\
\hline \multirow[t]{3}{*}{ Matsushita et al. [29] ${ }^{\mathrm{a}}$} & Case-control & Hospital/hospital & $70.2 \pm 10.0 / 70.1 \pm 10.1$ & $39.3 / 39.3$ & Matching for age and sex \\
\hline & Case-control & Hospital/hospital & $69.0 \pm 9.3 / 64.8 \pm 15.4$ & $35.3 / 45.7$ & Stratification by HT \\
\hline & Cohort & Prospective cohort & $\geq 40$ & NA & NA \\
\hline Sun et al. [35] & Case-control & Hospital/hospital & $73.2 \pm 9.4 / 73.3 \pm 7.3$ & $44 / 45$ & SNP83, age, sex, BMI, DM, HT, HD \\
\hline Munshi et al. [33] & Case-control & Hospital/population & $48.5 \pm 16.3 / 47.0 \pm 17.8$ & $24.8 / 26.0$ & Matching for age and sex \\
\hline
\end{tabular}

$\mathrm{BMI}=$ Body mass index DM = diabetes mellitus; $\mathrm{HT}=$ hypertension; $\mathrm{HD}=$ heart disease $\mathrm{HCL}=$ hypercholesterolemia; $\mathrm{SBP}=$ systolic blood pressure; $\mathrm{DBP}=$ diastolic blood pressure; $\mathrm{HDL}=$ high-density lipoprotein cholesterol; $\mathrm{NA}=$ not available .

${ }^{\text {a } I n c l u d e d} 2$ case-control studies and 1 cohort study.

to differences in population characteristics may dilute the true effect of a variant to the susceptibility of a disease), we calculated pooled ORs for homogenous substudies in the event that significant heterogeneity was observed [25]. We drew forest plots to summarize the ORs and 95\% CI for each meta-analysis showing a statistically significant association [23]. In addition, we examine funnel plots to find evidence of publication bias and used the rank correlation method for testing asymmetry $[23,27]$.

\section{Results}

We initially performed meta-analyses between each of the 6 SNPs reported in the Icelandic study and ischemic stroke. The subjects included in our meta-analysis consisted of 11,834 cases and 15,233 controls. Each of the original studies analyzed different numbers of study subjects and SNPs (table 1). While the study populations of most studies ( $\mathrm{n}=15)$ were of European descent, as in the original Icelandic study, 8 studies were from Japan, Pakistan, Taiwan, India, and China [28-35]. Table 2 summarizes the population characteristics and study designs of 23 studies included in subsequent analysis. We observed significant heterogeneity across studies with respect to ethnicity, study design, nonstandardized adjustments for modifiable and nonmodifiable risk factors for stroke, etc. Most studies recruited their case subjects from hospitals and their control subjects from the general population; however, 5 studies recruited case and control subjects from the general population of prospective cohort studies, and 3 studies obtained both groups of study subjects from hospitals. The mean age of subjects in most studies was within the range of 50-75 years, except in the studies by Munshi et al. [33] (i.e. cases, 48.5; controls, 47 years old) and Song et al. [36] (cases, 41.7; 
Table 3. Genotype frequencies for each of the 6 SNPs observed in the original publications

\begin{tabular}{|c|c|c|c|c|c|c|c|}
\hline \multicolumn{2}{|l|}{ Study } & \multirow{2}{*}{$\begin{array}{l}\text { Cases } \\
\text { TT/CT/CC }\end{array}$} & \multirow[t]{2}{*}{ Controls } & \multicolumn{2}{|l|}{ Study } & \multirow{2}{*}{$\begin{array}{l}\text { Cases } \\
\mathrm{CC} / \mathrm{CT} / \mathrm{TT}\end{array}$} & \multirow[t]{2}{*}{ Controls } \\
\hline SNP26 & $(\mathrm{rs} 40512)$ & & & SNP83 & $($ rs966221) & & \\
\hline & Gretarsdottir et al. [10] & $162 / 270 / 95$ & $118 / 203 / 72$ & & Gretarsdottir et al. [10] & $167 / 249 / 94$ & $98 / 167 / 84$ \\
\hline & Bevan et al. [11] & $258 / 338 / 138$ & $300 / 441 / 170$ & & Banerjee et al. [32] & $32 / 49 / 31$ & $38 / 110 / 64$ \\
\hline & Brophy et al. [37] & $80 / 122 / 46$ & $192 / 272 / 96$ & & Kuhlenbaumer et al. [41] & $434 / 546 / 179$ & $595 / 700 / 254$ \\
\hline & Lohmussaar et al. [12] & $176 / 339 / 107$ & $244 / 374 / 108$ & & Meschia et al. [39] & $164 / 139 / 55$ & $90 / 120 / 46$ \\
\hline & Zee et al. [38] & $91 / 123 / 45$ & 78/134/47 & & Nakayama et al. [28] & $3 / 42 / 163$ & $4 / 56 / 210$ \\
\hline \multirow{15}{*}{ SNP45 } & $(\mathrm{rs} 12188950)$ & CC/CT/TT & & & Saleheen et al. [30] & $55 / 96 / 47$ & $49 / 139 / 69$ \\
\hline & Gretarsdottir et al. [10] & $488 / 216 / 19$ & $300 / 167 / 25$ & & Song et al. [36] & $40 / 93 / 59$ & $45 / 93 / 48$ \\
\hline & Bevan et al. $[11]$ & $529 / 184 / 21$ & $659 / 240 / 29$ & & Staton et al. [13] & $44 / 68 / 39$ & $61 / 75 / 28$ \\
\hline & Brophy et al. [37] & $176 / 66 / 6$ & $406 / 142 / 12$ & & van Rijn et al. [42] & $34 / 37 / 17$ & $70 / 86 / 32$ \\
\hline & Fidani et al. [45] & $68 / 25 / 4$ & $72 / 28 / 2$ & & Woo et al. [40] & $108 / 170 / 68$ & $97 / 134 / 46$ \\
\hline & Kuhlenbaumer et al. [41] & $825 / 301 / 31$ & $1,140 / 394 / 29$ & & Lin et al. [31] & 6/54/117 & $13 / 51 / 147$ \\
\hline & Kostulas et al. [44] & $276 / 89 / 8$ & $\begin{array}{r}1,140 / 394 / 29 \\
545 / 188 / 18\end{array}$ & & Xue et al. [34] & $27 / 144 / 253$ & $29 / 255 / 603$ \\
\hline & Lohmussaar et al. [12] & $441 / 140 / 14$ & $\begin{array}{l}545 / 188 / 18 \\
550 / 167 / 17\end{array}$ & & Matsushita et al. [29] & $11 / 167 / 561$ & $85 / 910 / 2,734$ \\
\hline & Meschia et al. [39] & $186 / 165 / 25$ & $113 / 120 / 29$ & & Sun et al. [35] & $40 / 223 / 385$ & $35 / 230 / 496$ \\
\hline & Nilsson-Ardnor et al. [43] & $164 / 50 / 6$ & $342 / 94 / 6$ & & Munshi et al. [33] & $26 / 124 / 100$ & $5 / 100 / 145$ \\
\hline & Song et al. [36] & $143 / 48 / 4$ & $143 / 41 / 3$ & SNP87 & (rs2910829) & $\mathrm{CC} / \mathrm{CT} / \mathrm{TT}$ & \\
\hline & Staton et al. [13] & $106 / 38 / 6$ & $121 / 41 / 2$ & & Gretarsdottir et al. [10] & $148 / 315 / 179$ & $156 / 290 / 137$ \\
\hline & van Rijn et al. [42] & $65 / 19 / 5$ & $139 / 47 / 5$ & & Bevan et al. [11] & $154 / 360 / 212$ & $214 / 464 / 245$ \\
\hline & Zee et al. [38] & $178 / 73 / 8$ & $192 / 64 / 3$ & & Kuhlenbaumer et al. [41] & $216 / 505 / 293$ & $353 / 759 / 452$ \\
\hline & Lövkvist et al. [16] & $702 / 209 / 18$ & $271 / 111 / 13$ & & Lohmussaar et al. [12] & $128 / 296 / 174$ & $146 / 366 / 216$ \\
\hline \multirow[t]{15}{*}{ SNP56 } & (rs702553) & AA/AT/TT & & & Saleheen et al. [30] & $76 / 57 / 37$ & $86 / 78 / 39$ \\
\hline & Gretarsdottir et al. [10] & $52 / 211 / 287$ & $66 / 292 / 257$ & & Staton et al. [13] & $45 / 72 / 34$ & $36 / 72 / 56$ \\
\hline & Brophy et al. [37] & $30 / 113 / 105$ & $62 / 249 / 249$ & & Woo et al. [40] & 80/175/97 & $58 / 134 / 76$ \\
\hline & Kuhlenbaumer et al. [41] & $123 / 463 / 545$ & $157 / 653 / 738$ & & Lövkvist et al. [16] & $187 / 473 / 269$ & $72 / 208 / 114$ \\
\hline & & $68 / 151 / 136$ & $15 / / 653 / 138$ & & Lin et al. [31] & $120 / 52 / 8$ & $149 / 54 / 7$ \\
\hline & Staton et al. [13] & $\begin{array}{l}08 / 151 / 136 \\
27 / 66 / 58\end{array}$ & $\begin{array}{l}67 / 90 / 97 \\
21 / 78 / 65\end{array}$ & & Xue et al. [34] & $12 / 119 / 293$ & $26 / 257 / 604$ \\
\hline & Woo et al. [40] & $64 / 170 / 111$ & $\begin{array}{l}21 / 78 / 65 \\
39 / 131 / 114\end{array}$ & & Matsushita et al. [29] & $826 / 248 / 18$ & $2,840 / 950 / 57$ \\
\hline & Zee et al. [38] & $32 / 122 / 105$ & $\begin{array}{l}39 / 131 / 114 \\
20 / 118 / 121\end{array}$ & & Sun et al. [35] & $439 / 182 / 25$ & $539 / 202 / 20$ \\
\hline & Lin et al. [31] & $60 / 80 / 44$ & $90 / 87 / 33$ & SNP89 & $($ rs1396476) & $\mathrm{TT} / \mathrm{GT} / \mathrm{GG}$ & \\
\hline & Matsushita et al. [29] & $346 / 549 / 186$ & $1,185 / 1,872 / 771$ & & Gretarsdottir et al. [10] & $411 / 159 / 5$ & $295 / 140 / 15$ \\
\hline & Sun et al. [35] & $212 / 309 / 117$ & $241 / 379 / 139$ & & Kuhlenbaumer et al. [41] & $813 / 301 / 32$ & $1,093 / 432 / 39$ \\
\hline & & & & & Song et al. [36] & $140 / 49 / 4$ & $146 / 37 / 3$ \\
\hline & & & & & Staton et al. [13] & $100 / 45 / 6$ & $127 / 16 / 21$ \\
\hline & & & & & Woo et al. [40] & $237 / 88 / 9$ & $204 / 65 / 5$ \\
\hline & & & & & Matsushita et al. [29] & $989 / 103 / 2$ & $3,519 / 329 / 11$ \\
\hline
\end{tabular}
[10].

Genotypes were determined according to the allelic designation and allele frequencies of the Icelandic study by Gretarsdottir et al.

controls, 39.6 years old). In terms of gender composition, most studies were composed of about $25-70 \%$ women; however, Brophy et al. [37] and Song et al. [36] studied only women, while Zee et al. [38] included only men. Controlled variables differed across studies; Meschia et al. [39] and Woo et al. [40] adjusted for various variables, including smoking, hypertension, diabetes mellitus, and hypercholesterolemia; however, some studies did not provide any information on whether any variables were controlled for statistical analyses.

Meta-Analysis of the PDE4D Gene and Ischemic Stroke
We summarize the genotype frequencies for each of the 6 SNPs by case-control status obtained from the 23 original publications that were included in our metaanalysis (table 3). The reference genotypes for all studies were determined according to those of the Icelandic study. Genotype distributions of SNP83 in the case and control groups of 8 studies which are composed mostly of Asian populations seemed different from those of 6 other studies in Caucasians [28-35]. The ORs and 95\% CI of previous studies were not directly comparable because

Neuroepidemiology 2011;36:213-222 
Table 4. Results of the meta-analysis under 3 genetic models and the test for heterogeneity of published studies that examined the association between each of 6 SNPs and ischemic stroke

\begin{tabular}{|c|c|c|c|c|c|c|c|c|}
\hline \multirow{2}{*}{$\begin{array}{l}\text { SNPs } \\
(\mathrm{M} / \mathrm{m})^{\mathrm{a}}\end{array}$} & \multirow{2}{*}{$\begin{array}{l}\text { Studies } \\
\mathrm{n}\end{array}$} & \multirow{2}{*}{$\begin{array}{l}\text { Cases, } \mathrm{n} \\
\text { Controls, } \mathrm{n}\end{array}$} & \multicolumn{2}{|c|}{ Dominant $^{\mathrm{b}}$ (carrier) } & \multicolumn{2}{|c|}{ Recessive (homozygote) ${ }^{\mathrm{b}}$} & \multicolumn{2}{|c|}{ Codominant (heterozygote/homozygote) ${ }^{\mathrm{b}}$} \\
\hline & & & OR $(95 \% \mathrm{CI})^{\mathrm{c}}$ & $\mathrm{I}^{2}(\mathrm{p})^{\mathrm{c}}$ & OR $(95 \% \mathrm{CI})$ & $\mathrm{I}^{2}(\mathrm{p})$ & OR (95\% CI) & $\mathrm{I}^{2}(\mathrm{p})$ \\
\hline $\begin{array}{l}\text { SNP26 } \\
\text { rs40512 } \\
(\mathrm{T} / \mathrm{C})\end{array}$ & 5 & $\begin{array}{l}\text { TT/CT/CC } \\
767 / 1,192 / 431 \\
932 / 1,424 / 493\end{array}$ & $\begin{array}{l}1.01 \\
(0.85-1.18)\end{array}$ & $\begin{array}{l}43.60 \\
(0.131)\end{array}$ & $\begin{array}{l}1.05 \\
(0.90-1.21)\end{array}$ & $\begin{array}{l}0.00 \\
(0.877)\end{array}$ & $\begin{array}{l}0.99 / 1.05 \\
(0.85-1.17) /(0.88-1.24)\end{array}$ & $\begin{array}{l}34.80 / 8.40 \\
(0.189) /(0.358)\end{array}$ \\
\hline $\begin{array}{l}\text { SNP45 } \\
\text { rs12188950 } \\
(\mathrm{C} / \mathrm{T})\end{array}$ & 14 & $\begin{array}{l}\text { CC/CT/TT } \\
4,347 / 1,623 / 175 \\
4,993 / 1,844 / 193 \\
2,971 / 1,033 / 113 \\
4,309 / 1,446 / 126\end{array}$ & $\begin{array}{l}0.96 \\
(0.87-1.06) \\
1.05 \\
(0.96-1.15)\end{array}$ & $\begin{array}{l}31.50 \\
(0.123) \\
0.00 \\
(0.958)\end{array}$ & $\begin{array}{l}1.01 \\
(0.75-1.36) \\
1.28 \\
(0.98-1.67)\end{array}$ & $\begin{array}{l}38.60 \\
(0.069) \\
0.00 \\
(0.719)\end{array}$ & $\begin{array}{l}0.96 / 1.01 \\
(0.88-1.04) /(0.74-1.39) \\
1.03 / 1.29 \\
(0.94-1.13) /(0.99-1.68)\end{array}$ & $\begin{array}{l}0.00 / 45.90 \\
(0.530) /(0.031)^{c} \\
0.00 / 0.00 \\
(0.989) /(0.691)\end{array}$ \\
\hline $\begin{array}{l}\text { SNP56 } \\
\text { rs702553 } \\
(\mathrm{A} / \mathrm{T})\end{array}$ & 9 & $\begin{array}{l}\mathrm{AA} / \mathrm{AT} / \mathrm{TT} \\
1,678 / 2,023 / 691 \\
2,900 / 3,657 / 1,309 \\
1,332 / 1,474 / 505 \\
1,715 / 1,785 / 538\end{array}$ & $\begin{array}{l}1.05 \\
(0.95-1.17) \\
1.11 \\
(0.96-1.27)\end{array}$ & $\begin{array}{l}29.60 \\
(0.181) \\
37.70 \\
(0.141)\end{array}$ & $\begin{array}{l}1.08 \\
(0.89-1.32) \\
1.19 \\
(1.02-1.39) \\
\mathrm{p}=0.022\end{array}$ & $\begin{array}{l}61.80 \\
(0.007) \\
8.60 \\
(0.362)\end{array}$ & $\begin{array}{l}1.03 / 1.13 \\
(0.94-1.12) /(0.91-1.41) \\
1.03 / 1.29 \\
(0.93-1.14) /(1.03-1.61) \\
\mathrm{p}=0.022\end{array}$ & $\begin{array}{l}0.00 / 63.10 \\
(0.560) /(0.005)^{\mathrm{c}} \\
0.70 / 41.70 \\
(0.418) /(0.112)\end{array}$ \\
\hline $\begin{array}{l}\text { SNP83 } \\
\text { rs966221 } \\
(\mathrm{C} / \mathrm{T})\end{array}$ & 10 & $\begin{array}{l}\mathrm{CC} / \mathrm{CT} / \mathrm{TT} \\
1,024 / 1,952 / 2,074 \\
1,216 / 3,059 / 4,922 \\
872 / 1,520 / 1,635 \\
1,072 / 2,490 / 4,077 \\
163 / 608 / 996 \\
168 / 841 / 1,589\end{array}$ & $\begin{array}{l}0.85 \\
(0.68-1.07) \\
0.95 \\
(0.77-1.16) \\
0.66 \\
(0.50-0.87) \\
p=0.0034\end{array}$ & $\begin{array}{l}69.40 \\
\left(1 \times 10^{-4}\right) \\
51.50 \\
(0.029) \\
22.40 \\
(0.265)\end{array}$ & $\begin{array}{l}0.92 \\
(0.78-1.06) \\
0.97 \\
(0.87-1.09) \\
0.79 \\
(0.69-0.90) \\
p=0.0005\end{array}$ & $\begin{array}{l}63.30 \\
(0.0007) \\
19.70 \\
(0.261) \\
0.00 \\
(0.711)\end{array}$ & $\begin{array}{l}0.87 / 0.86 \\
(0.71-1.06) /(0.65-1.13) \\
0.95 / 0.98 \\
(0.77-1.16) /(0.78-1.21) \\
0.72 / 0.64 \\
(0.53-0.99) /(0.48-0.85) \\
\mathrm{p}=0.046 / 0.0025\end{array}$ & $\begin{array}{l}58.10 / 70.30 \\
(0.003) /\left(1 \times 10^{-4}\right)^{c} \\
45.00 / 37.50 \\
(0.059) /(0.109) \\
31.00 / 16.50 \\
(0.203) /(0.307)\end{array}$ \\
\hline $\begin{array}{l}\text { SNP87 } \\
\text { rs2910829 } \\
\text { (C/T) } \\
\text { Asian studies }\end{array}$ & 11 & $\begin{array}{l}\text { CC/CT/TT } \\
2,283 / 2,539 / 1,460 \\
4,519 / 3,544 / 1,886 \\
1,473 / 658 / 381 \\
3,640 / 1,541 / 727\end{array}$ & $\begin{array}{l}0.992 \\
(0.91-1.07) \\
0.98 \\
(0.88-1.11)\end{array}$ & $\begin{array}{l}0.00 \\
(0.539) \\
0.00 \\
(0.427)\end{array}$ & $\begin{array}{l}1.02 \\
(0.93-1.11) \\
1.12 \\
(0.92-1.35)\end{array}$ & $\begin{array}{l}0.00 \\
(0.518) \\
0.00 \\
(0.860)\end{array}$ & $\begin{array}{l}0.98 / 1.02 \\
(0.90-1.06) /(0.90-1.15) \\
0.96 / 1.18 \\
(0.85-1.08) /(0.89-1.57)\end{array}$ & $\begin{array}{l}0.00 / 3.90 \\
(0.795) /(0.405) \\
0.00 / 0.00 \\
(0.495) /(0.883)\end{array}$ \\
\hline $\begin{array}{l}\text { SNP89 } \\
\text { rs1396476 } \\
(\mathrm{T} / \mathrm{G})\end{array}$ & 4 & $\begin{array}{l}\text { TT/GT/GG } \\
2,279 / 586 / 53 \\
5,089 / 879 / 79 \\
2,179 / 541 / 47 \\
4,962 / 863 / 58\end{array}$ & $\begin{array}{l}1.14 \\
(0.95-1.37) \\
1.05 \\
(0.92-1.19)\end{array}$ & $\begin{array}{l}44.50 \\
(0.125) \\
8.70 \\
(0.349)\end{array}$ & $\begin{array}{l}0.82 \\
(0.43-1.55) \\
1.13 \\
(0.75-1.69)\end{array}$ & $\begin{array}{l}50.10 \\
(0.091) \\
0.00 \\
(0.845)\end{array}$ & $\begin{array}{l}1.29 / 0.89 \\
(0.95-1.75) /(0.53-1.50) \\
1.04 / 1.12 \\
(0.91-1.20) /(0.75-1.69)\end{array}$ & $\begin{array}{l}77.50 / 29.00 \\
(0.001)^{c} /(0.228) \\
12.00 / 0.00 \\
(0.332) /(0.820)\end{array}$ \\
\hline
\end{tabular}

ORs and $95 \%$ CIs were computed using random effects models. The $\mathrm{p}$ value was listed below OR if $\mathrm{p}$ was $<0.05$. $\mathrm{I}^{2}=$ Heterogeneity $(\%)$; $(\mathrm{p})=\mathrm{p}$ value for heterogeneity ( $Q$ statistics).

${ }^{a}$ Major/minor alleles were determined by the allele frequency reported by Gretardottir et al. [10].

${ }^{\mathrm{b}}$ Genetic model: dominant, $\mathrm{MM}$ vs. $\mathrm{Mm}+\mathrm{mm}$; recessive, $\mathrm{MM}+\mathrm{Mm}$ vs. mm, and codominant, heterozygote $\mathrm{MM}$ vs. Mm, homozygote $\mathrm{MM}$ vs. mm.

c Subgroup analysis was performed in the genetic model being marked.

the statistical models and/or genetic models used in each study were different; furthermore, adjusted covariates varied across studies as shown in table 2.

We performed a meta-analysis using fixed- and random-effect models comprised of 23 independent studies. The analysis proceeded excluding original studies due to the variability introduced by a stronger genetic effect of the first study compared to those subsequent studies showing less significance [36, 37]. Table 4 lists the pooled genotypic ORs and the 95\% CI for each SNP, as determined using 3 genetic models (estimates with the fixed effects models are not shown). After sensitivity analyses excluding the studies responsible for the significant heterogeneity $\left(\mathrm{I}^{2}\right)$, we performed a meta-analysis on the group of homogenous studies [26]. Heterogeneity among studies was determined using the $\mathrm{I}^{2}>50 \%$ or Q statistics $\mathrm{p}<0.1$. We present the pooled ORs of homogeneous substudies.

In the meta-analysis, we failed to find a significant association of SNP26 with ischemic stroke. For SNP45, we performed the initial meta-analysis using 14 studies and subsequently identified significant heterogeneity in the 
Fig. 2. Forest (a) and funnel (b) plots for SNP56 after removing the studies responsible for significant heterogeneity in the meta-analysis. The first author of each study is presented in $\mathbf{a}$ and $\mathbf{b}$.
Fig. 3. Forest (a) and funnel (b) plots for SNP83 using Asian studies after removing the studies responsible for significant heterogeneity in the meta-analysis. The first author of each study is presented in a and $\mathbf{b}$.
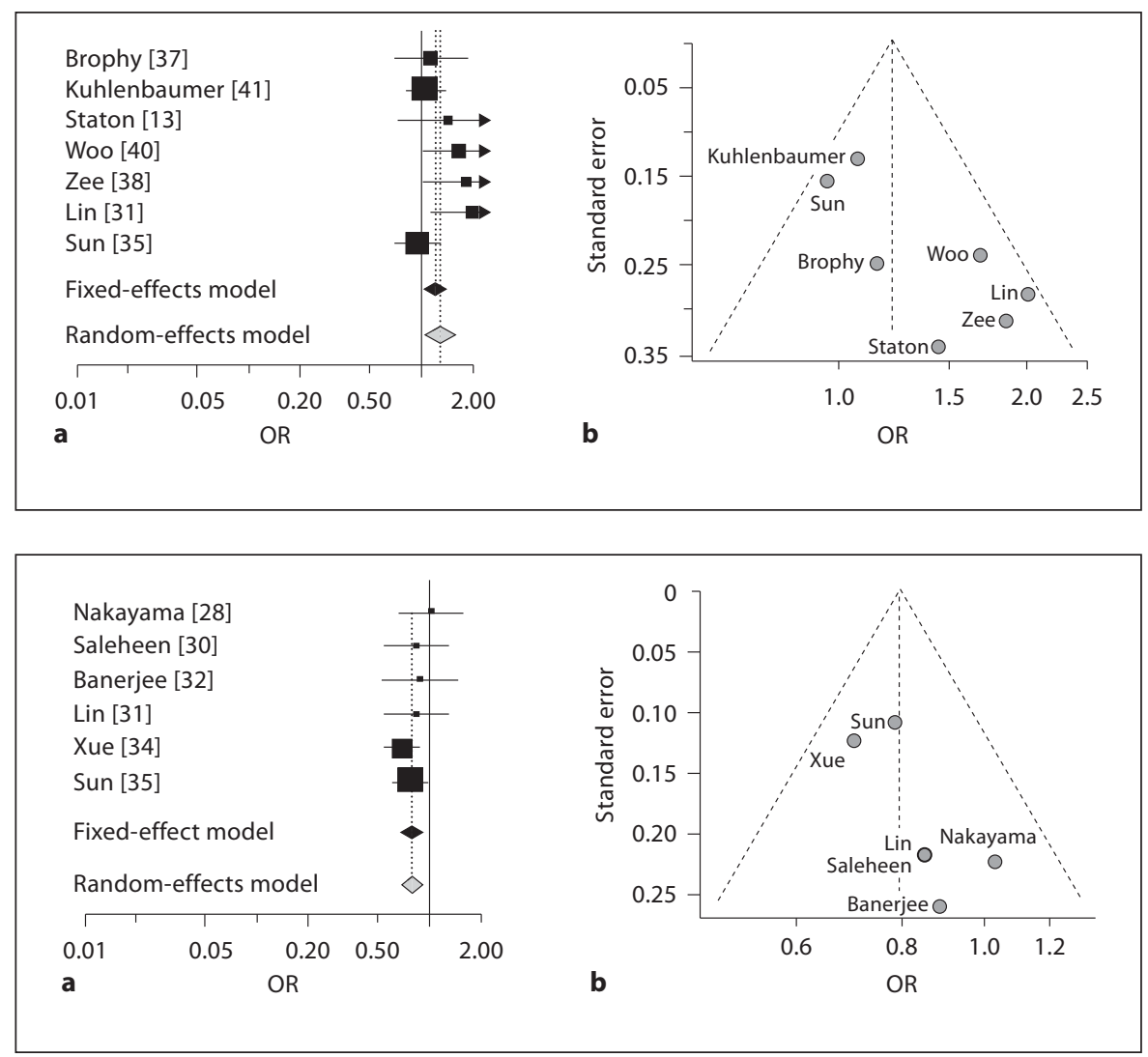

codominant model. Sensitivity analysis identified that 3 studies (i.e. Gretarsdottir et al. [10], Lövkvist et al. [16], and Meschia et al. [39]) introduced considerable heterogeneity. After removing these 3 studies from the subsequent metaanalysis of 11 studies, we observed a marginally significant overall OR in the TT homozygote group under the codominant model $(\mathrm{OR}=1.29,95 \% \mathrm{CI}=0.99-1.68 ; \mathrm{p}=0.05)$.

The major allele of SNP56 in the Icelandic population was allele $\mathrm{A}$, and the genotype frequencies included in the meta-analysis were 1,678/2,023/691 and 2,900/3,657/1,309 for case and control groups, respectively. The interstudy heterogeneity was introduced by the studies of Matsushita et al. [29] and Meschia et al. [39] in the meta-analysis of 9 studies $\left(\mathrm{I}^{2}=63.10 \% ; \mathrm{p}=0.005\right)$. After removing these studies from the subsequent meta-analysis, we observed the most significant overall OR in a codominant model by comparison of individuals with the TT genotype to the AA reference group $(\mathrm{OR}=1.29,95 \% \mathrm{CI}=$ $1.03-1.61 ; \mathrm{p}=0.022)$. However, a recessive mode of inheritance might be a better fit to SNP56 because the OR for the AT heterozygous group was close to the baseline value of 1 in the codominant model. Figure 2a illustrates the ORs and 95\% CIs of individual studies, as well as the overall ORs of the SNP56 in each of the fixed- and random-effects models. As shown in figure $2 b$, neither interstudy heterogeneity nor publication bias for the SNP56 seemed to exist anymore $\left(\mathrm{I}^{2}=41.70 \% ; \mathrm{p}=0.112\right)$.

In the case of SNP83, four different studies were excluded from the analysis due to heterogeneity and possible publication bias (i.e. Staton et al. [13], Saleheen et al. [30], Munshi et al. [33], and Xue et al. [34]). However, the ORs estimated in 10 homogenous studies were close to 1. Since the genotype frequencies of SNP83 were different by ethnicity, we performed ethnic-specific meta-analyses in Asian populations [28, 30-32, 34, 35] and non-Asian populations $[4,13,36,40-42]$, respectively. The studies of Matsushita et al. [29] and Munshi et al. [33] were excluded from the Asian subgroup analysis because of its large heterogeneity estimated in the sensitivity analysis. A protective effect of the rare $\mathrm{T}$ allele was observed in Asian populations and the effect seems to best fit the recessive genetic model $(\mathrm{OR}=0.79,95 \% \mathrm{CI}=0.69-0.90 ; \mathrm{p}=0.0005)$ (fig. 3a). No significant association was observed in nonAsian populations (data not shown). 
For SNP87, eleven studies did not show any significant association. We performed a meta-analysis in Asian groups (i.e. Matsushita et al. [29], Saleheen et al. [30], Lin et al. [31], Xue et al. [34], and Sun et al. [35]) and found no evidence of a genetic association of SNP87 with ischemic stroke. After removing Staton et al. [13] from the initial analysis, the heterogeneity of SNP89 significantly decreased. However, the effect size was close to null.

\section{Discussion}

The current meta-analysis consisted of 11,834 cases and 15,233 controls. In this meta-analysis, we included 7 more studies (i.e. Lövkvist et al. [16], Matsushita et al. [29], Lin et al. [31], Banerjee et al. [32], Munshi et al. [33], Xue et al. [34], and Sun et al. [35]) published after the study by Bevan et al. [17]. Among the 6 SNPs analyzed, our metaanalysis of 7 homogenous studies showed a significant association between SNP56 and ischemic stroke $(\mathrm{p}=0.022)$. The result showed that individuals with the SNP56 TT genotype were $29 \%$ more likely to develop ischemic stroke compared with the common AA genotype under the random-effects model. In terms of ethnic difference, a protective effect of the ancestral T allele at SNP83 was observed only in Asian populations (ORTT $=0.79 ; \mathrm{p}=$ 0.0005). This result is in agreement with those of Xu et al. [19].

The reversed direction of the effects of PDE4D gene variants observed in different studies suggested the possibility of a spurious association detected in Icelanders or in other study populations. It is important to note that some studies designated alleles based on the $3^{\prime}$-to- $5^{\prime}$ orientation of the gene, while other studies followed the $5^{\prime}$ to- $3^{\prime}$ orientation on the chromosome. For instance, the allele of SNP45 was reported as C/T in 4 (i.e. Bevan et al. [11], Lövkvist et al. [16], Song et al. [36], and van Rijin et al. [42]) of 14 studies but was reported as A/G in the rest of the studies, including the Icelandic study. Thus, the allelic designation for an SNP must be taken into consideration when the result of the SNP is compared across studies. In addition, a major genotype that is conventionally used as a reference genotype in genetic association studies may not always be the same among different ethnic populations as in the case of SNP83. In the current study, the summarized OR was computed from genotypic frequencies, and the reference genotypes were consistently determined for all studies according to the allelic designation and allele frequencies of the Icelandic study.
Heterogeneity across studies may have confounded or diluted the overall estimate of the meta-analysis if conducted with all of these studies without any consideration for incomparability. In the current study, the OR for SNP83 for all of the studies combined was close to 1, while it was 0.79 in Asian studies. The frequency of the $\mathrm{C}$ allele in Asians (i.e. 0.156 for JPT and 0.211 for HCB) is significantly different from that in Europeans (i.e. 0.592). The difference in allele frequency between Asian and European populations and the meta-analysis of Asian studies indicates that SNP83 may affect the risk of ischemic stroke in an Asian-specific manner. Bevan et al. [17] performed their meta-analysis only in the basic fixed-effects model without consideration of significant heterogeneity. It might be responsible for the failure of this study to replicate previous findings. The analysis strategy considering the design of each study would be another critical factor for removing spurious findings [41]. For instance, Matsushita et al. [29] reported that significant association of SNP56 in the prospective cohort, while they failed to detect the association in 2 case-control studies. Thus, careful consideration of multiple confounding factors and heterogeneity among studies may be necessary to perform a meta-analysis.

Some important concerns are discussed below. Firstly, different allele frequencies across study populations, particularly in different ethnic groups, might cause inconsistency among research findings and may result in a loss of statistical power. Secondly, stratification analysis by factors causing heterogeneity across studies may be necessary to reveal more reliable information. Due to the limited number of studies available for this meta-analysis, we could not perform separate meta-analyses for case-control studies and prospective cohort studies, which may differ in various aspects. Finally, confounding variables (e.g. age and sex) were not adjusted in the meta-analysis because covariate data were not given in most references. Nonetheless, this meta-analysis included the most studies published so far on the genetic association of $P D E 4 D$ gene variants and ischemic stroke. In the presence of significant heterogeneity, influence analysis objectively explored all combinations of studies to achieve homogeneity and to prevent heterogeneity from having influence across studies in the overall effect estimate at a given SNP. The genotypic frequencies of previous studies were obtained from the authors of each study if only allelic frequencies were shown in the publication, and the accuracies of the genotypic frequencies and configurations were thoroughly checked before combining the data from all of the studies. Owing to the complexity of finding suit- 
able genetic models for SNPs, the association of each SNP was tested under 3 different genetic models, allowing for heterogeneity across studies.

Insignificant results observed in SNPs other than SNP56 and SNP83 do not rule out a true association of any of the reported variants with ischemic stroke, particularly in the case of SNP45. In consideration of the issues discussed above, applications of valid meta-analytic methods along with continuous efforts to combine the results of the growing number of related studies may allow investigators to confirm the true effects of the PDE $4 D$ gene with ischemic stroke in different ethnic groups.

\section{Acknowledgements}

This study was supported by the Center for Genome Science, Republic of Korea, (National Institute of Health research contract 2007-E00004-00). We thank Dr. Lövkvist, who provided genotype data, and we thank Dr. Daniel Woo, Dr. James F. Meschia, Dr. Robert Y.L. Zee, Dr. J. M. Staton, Dr. John W. Eikelboom, and Dr. Steve Bevan for helpful discussions.

\section{Disclosure Statement}

The authors declare no conflict of interest.

\section{References}

1 Strong K, Mathers C, Bonita R: Preventing stroke: saving lives around the world. Lancet Neurol 2007;6:182-187.

2 Rosand J, Bayley N, Rost N, de Bakker PI: Many hypotheses but no replication for the association between PDE4D and stroke. Nat Genet 2006;38:1091-1092; author reply 1092-1093.

-3 Goldstein LB, Adams R, Alberts MJ, Appel LJ, Brass LM, Bushnell CD, Culebras A, DeGraba TJ, Gorelick PB, Guyton JR, Hart RG, Howard G, Kelly-Hayes M, Nixon JV, Sacco RL: Primary prevention of ischemic stroke: a guideline from the American Heart Association/American Stroke Association Stroke Council - cosponsored by the Atherosclerotic Peripheral Vascular Disease Interdisciplinary Working Group; Cardiovascular Nursing Council; Clinical Cardiology Council; Nutrition, Physical Activity, and Metabolism Council; and the Quality of Care and Outcomes Research Interdisciplinary Working Group: The American Academy of Neurology affirms the value of this guideline. Stroke 2006;37:1583-1633.

$\checkmark 4$ Meschia JF: Subtyping in ischemic stroke genetic research. J Stroke Cerebrovasc Dis 2002;11:208-219.

5 Dichgans M, Markus HS: Genetic association studies in stroke: methodological issues and proposed standard criteria. Stroke 2005; 36:2027-2031.

6 Touze E, Rothwell PM: Sex differences in heritability of ischemic stroke: a systematic review and meta-analysis. Stroke 2008;39: 16-23.

7 Schulz UG, Flossmann E, Rothwell PM: Heritability of ischemic stroke in relation to age, vascular risk factors, and subtypes of incident stroke in population-based studies. Stroke 2004;35:819-824.

8 Moskau S, Golla A, Grothe C, Boes M, Pohl C, Klockgether T: Heritability of carotid artery atherosclerotic lesions: an ultrasound study in 154 families. Stroke 2005;36:5-8.
> Bolger GB, Erdogan S, Jones RE, Loughney K, Scotland G, Hoffmann R, Wilkinson I, Farrell C, Houslay MD: Characterization of five different proteins produced by alternatively spliced mRNAs from the human camp-specific phosphodiesterase PDE4D gene. Biochem J 1997;328:539-548

10 Gretarsdottir S, Thorleifsson G, Reynisdottir ST, Manolescu A, Jonsdottir S, Jonsdottir T, Gudmundsdottir T, Bjarnadottir SM, Einarsson OB, Gudjonsdottir HM: The gene encoding phosphodiesterase $4 \mathrm{D}$ confers risk of ischemic stroke. Nat Genet 2003;35:131-138.

- 11 Bevan S, Porteous L, Sitzer M, Markus HS Phosphodiesterase 4D gene, ischemic stroke, and asymptomatic carotid atherosclerosis. Stroke 2005;36:949-953.

12 Lohmussaar E, Gschwendtner A, Mueller JC, Org T, Wichmann E, Hamann G, Meitinger T, Dichgans M: ALOX5AP gene and the PDE4D gene in a central European population of stroke patients. Stroke 2005;36:731736.

13 Staton JM, Sayer MS, Hankey GJ, Attia J, Thakkinstian A, Yi Q, Cole VJ, Baker R, Eikelboom JW: Association between phosphodiesterase 4D gene and ischaemic stroke. J Neurol Neurosurg Psychiatry 2006;77: 1067-1069.

14 Worrall BB, Mychaleckyj JC: PDE4D and stroke: a real advance or a case of the emperor's new clothes? Stroke 2006;37:19551957.

15 Berlin JA, Kim CJ: The use of meta-analysis in pharmacoepidemiology; in Strom BL (ed): Pharmacoepidemiology, ed 4. New York, Wiley, 2007, pp 681-707.

16 Lövkvist H, Smith JG, Luthman H, Hoglund P, Norrving B, Kristoffersson U, Jonsson A-C, Lindgren AG: Ischaemic stroke in hypertensive patients is associated with variations in the PDE4D genome region. Eur Hum Genet 2008;16:1117-1125.
17 Bevan S, Dichgans M, Gschwendtner A, Kuhlenbaumer G, Ringelstein EB, Markus HS: Variation in the PDE4D gene and ischemic stroke risk: a systematic review and meta-analysis on 5,200 cases and 6,600 controls. Stroke 2008;39:1966-1971.

$\checkmark 18$ Domingues-Montanari S, Fernández-Cadenas I, del Rio-Espinola A, Corbeto N, Krug T, Manso H, Gouveia L, Sobral J, Mendioroz M, Fernández-Morales J, Alvarez-Sabin J, Ribó M, Rubiera M, Obach V, Martí-Fàbregas J, Freijo M, Serena J, Ferro JM, Vicente AM, Oliveira SA, Montaner J: Association of a genetic variant in the ALOX5AP with higher risk of ischemic stroke: a case-control, meta-analysis and functional study. Cerebrovasc Dis 2010;29:528-537.

19 Xu X, Li X, Li J, Ou R, Sheng W: Meta-anal$y$ sis of association between variation in the PDE4D gene and ischemic cerebral infarction risk in Asian populations. Neurogenetics 2010;11:327-333.

20 Conti M, Richter W, Mehats C, Livera G, Park JY, Jin C: Cyclic AMP-specific PDE4 phosphodiesterases as critical components of cyclic AMP signaling. J Biol Chem 2003; 278:5493-5496.

21 Hakonarson H: Role of FLAP and PDE4D in myocardial infarction and stroke: target discovery and future treatment options. Curr Treat Options Cardiovasc Med 2006;8:183192.

$>22$ DerSimonian R, Laird N: Meta-analysis in clinical trials. Control Clin Trials 1986;7: 177-188.

23 Sutton AJ, Abrams KR, Jones DR, Sheldon TA, Song F: Methods for meta-analysis in medical research. New York, Wiley, 2000.

24 Higgins JP, Thompson SG, Deeks JJ, Altman DG: Measuring inconsistency in meta-analysis. BMJ 2003;327:557-560.

25 Higgins JP, Thompson SG: Quantifying heterogeneity in a meta-analysis. Stat Med 2002; 21:1539-1558. 
26 Patsopoulos NA, Evangelou E, Ioannidis JP: Sensitivity of between-study heterogeneity in meta-analysis: proposed metrics and empirical evaluation. Int J Epidemiol 2008;37: 1148-1157.

-27 Egger M, Smith GD, Schneider M, Minder C: Bias in meta-analysis detected by a simple, graphical test. BMJ 1997;315:629-634.

28 Nakayama T, Asai S, Sato N, Soma M: Genotype and haplotype association study of the STRK1 region on 5 q12 among Japanese: a case-control study. Stroke 2006;37:69-76.

29 Matsushita T, Kubo M, Yonemoto K, Ninomiya T, Ashikawa K, Liang B, Hata J, Doi Y, Kitazono T, Ibayashi S, Iida M, Kiyohara Y, Nakamura Y: Lack of association between variations of PDE4D and ischemic stroke in the Japanese population. Stroke 2009;40:1245-1251.

-30 Saleheen D, Bukhari S, Haider SR, Nazir A, Khanum S, Shafqat S, Anis MK, Frossard P: Association of phosphodiesterase 4D gene with ischemic stroke in a Pakistani population. Stroke 2005;36:2275-2277.

>31 Lin HF, Liao YC, Liou CW, Liu CK, Juo SH: The phosphodiesterase 4D gene for early onset ischemic stroke among normotensive patients. J Thromb Haemost 2007;5:436-438.

>32 Banerjee I, Gupta V, Ahmed T, Faizaan M, Agarwal P, Ganesh S: Inflammatory system gene polymorphism and the risk of stroke: a case-control study in an Indian population. Brain Res Bull 2008;75:158-165.

\33 Munshi A, Babu MS, Kaul S, Shafi G, Anila AN, Alladi S, Jyothy A: Phosphodiesterase 4D (PDE4D) gene variants and the risk of ischemic stroke in a South Indian population. J Neurol Sci 2009;285:142-145.
34 Xue H, Wang H, Song X, Li W, Sun K, Zhang W, Wang X, Wang Y, Hui R: Phosphodiesterase $4 \mathrm{D}$ gene polymorphism is associated with ischaemic and haemorrhagic stroke. Clin Sci (Lond) 2009;116:335-340.

35 Sun Y, Huang Y, Chen X, Liu Y, Lu X, Shi Y, Tang W, Yang J, Chen W, Zhao X, Gao L, Li S, Feng G, He L: Association between the PDE4D gene and ischaemic stroke in the Chinese Han population. Clin Sci (Lond) 2009; 117:265-272.

\$36 Song Q, Cole JW, O'Connell JR, Stine OC, Gallagher M, Giles WH, Mitchell BD, Wozniak MA, Stern BJ, Sorkin JD, McArdle PF, Naj AC, Xu Q, Gibbons GH, Kittner SJ: Phosphodiesterase 4D polymorphisms and the risk of cerebral infarction in a biracial population: the Stroke Prevention in Young Women Study. Hum Mol Genet 2006;15:24682478.

37 Brophy VH, Ro SK, Rhees BK, Lui LY, Lee JM, Umblas N, Bentley LG, Li J, Cheng S, Browner WS, Erlich HA: Association of phosphodiesterase 4D polymorphisms with ischemic stroke in a US population stratified by hypertension status. Stroke 2006;37: 1385-1390.

38 Zee RY, Brophy VH, Cheng S, Hegener HH, Erlich HA, Ridker PM: Polymorphisms of the phosphodiesterase 4D, cAMP-specific (PDE4D) gene and risk of ischemic stroke: a prospective, nested case-control evaluation. Stroke 2006;37:2012-2017.

39 Meschia JF, Brott TG, Brown RD Jr, Crook R, Worrall BB, Kissela B, Brown WM, Rich SS, Case LD, Evans EW, Hague S, Singleton A, Hardy J: Phosphodiesterase 4D and 5-lipoxygenase activating protein in ischemic stroke. Ann Neurol 2005;58:351-361.
40 Woo D, Kaushal R, Kissela B, Sekar P, Wolujewicz M, Pal P, Alwell K, Haverbusch M, Ewing I, Miller R, Kleindorfer D, Flaherty M, Chakraborty R, Deka R, Broderick J: Association of phosphodiesterase $4 \mathrm{D}$ with ischemic stroke: a population-based case-control study. Stroke 2006;37:371-376.

41 Kuhlenbaumer G, Berger K, Huge A, Lange E, Kessler C, John U, Funke H, Nabavi DG, Stogbauer F, Ringelstein EB, Stoll M: Evaluation of single nucleotide polymorphisms in the phosphodiesterase 4D gene (PDE4D) and their association with ischaemic stroke in a large German cohort. J Neurol Neurosurg Psychiatry 2006;77:521-524.

42 van Rijn MJ, Slooter AJ, Schut AF, Isaacs A, Aulchenko YS, Snijders PJ, Kappelle LJ, van Swieten JC, Oostra BA, van Duijn CM: Familial aggregation, the PDE4D gene, and ischemic stroke in a genetically isolated population. Neurology 2005;65:1203-1209.

43 Nilsson-Ardnor S, Wiklund PG, Lindgren P, Nilsson AK, Janunger T, Escher SA, Hallbeck B, Stegmayr B, Asplund K, Holmberg D: Linkage of ischemic stroke to the pde4d region on $5 \mathrm{q}$ in a Swedish population. Stroke 2005;36:1666-1671.

44 Kostulas K, Gretarsdottir S, Kostulas V, Manolescu A, Helgadottir A, Thorleifsson G, Gudmundsson LJ, Thorsteinsdottir U, Gulcher JR, Stefansson K, Hillert J: PDE4D and ALOX5AP genetic variants and risk for ischemic cerebrovascular disease in Sweden. J Neurol Sci 2007;263:113-117.

45 Fidani L, Clarimon J, Goulas A, Hatzitolios AI, Evans W, Tsirogianni E, Hardy J, Kotsis A: Association of phosphodiesterase $4 \mathrm{D}$ gene G0 haplotype and ischaemic stroke in a Greek population. Eur J Neurol 2007;14:745-749. 